\title{
Correction to the paper "Homogenization and Dispersion Effects in the Problem of Propagation of Waves Generated by a Localized Source" (Proc. Steklov Inst. Math. 281, 161-178 (2013))
}

\author{
V. V. Grushin ${ }^{a}$, S. Yu. Dobrokhotov ${ }^{b, c}$, and S. A. Sergeev ${ }^{b, c}$ \\ Received November 2014
}

DOI: $10.1134 / \mathrm{S} 0081543815010216$

Unfortunately, some erroneous signs were carried over into [1] from the earlier paper [2]. The following formulas should be corrected:

1. In formula (4.3) (p. 183 of the Russian original, and p. 174 of the English translation), the equality for $\widetilde{L}_{1}$ should have the form

$$
\widetilde{L}_{1}=-\frac{i \mu}{2}\left\langle\nabla_{x}, \nabla_{y}\right\rangle L_{0}-\left(\frac{\delta^{2}}{\mu} R(x, p)+\lambda M(x, p)\right) .
$$

2. In formula (4.5) (p. 183 of the Russian original, and p. 174 of the English translation), the equality for $\mathcal{L}_{1}$ should have the form

$$
\mathcal{L}_{1}=-\frac{i}{2}\left\langle\nabla_{x}, \nabla_{y}\right\rangle \mathcal{L}_{0}+\frac{1}{2 \mathcal{L}_{0}}\left(\frac{\delta^{2}}{\mu} R(x, p)+\lambda M(x, p)\right) .
$$

In both cases the sign of the second term on the right-hand side was wrong. As a result, the subsequent formulas in the derivation of the transport equation and in the transport equation itself are inaccurate to the same extent.

The conclusion on the existence of an anomalous dispersion in this case turns out to be false. Rapidly oscillating regions of the bottom give rise to effects that are qualitatively similar to those in the case of an ordinary dispersion.

\section{REFERENCES}

1. V. V. Grushin, S. Yu. Dobrokhotov, and S. A. Sergeev, "Homogenization and dispersion effects in the problem of propagation of waves generated by a localized source," Tr. Mat. Inst. im. V.A. Steklova, Ross. Akad. Nauk 281, 170-187 (2013) [Proc. Steklov Inst. Math. 281, 161-178 (2013)].

2. S. Yu. Dobrokhotov, S. A. Sergeev, and B. Tirozzi, "Asymptotic solutions of the Cauchy problem with localized initial conditions for linearized two-dimensional Boussinesq-type equations with variable coefficients," Russ. J. Math. Phys. 20 (2), 155-171 (2013).

\footnotetext{
${ }^{a}$ Moscow Institute of Electronics and Mathematics, National Research University Higher School of Economics, Bol'shoi Trekhsvyatitel'skii per. 3, Moscow, 109028 Russia.

${ }^{b}$ Institute for Problems in Mechanics, Russian Academy of Sciences, pr. Vernadskogo 101, korp. 1, Moscow, 119526 Russia.

${ }^{c}$ Moscow Institute of Physics and Technology (State University), Institutskii per. 9, Dolgoprudnyi, Moscow oblast, 141700 Russia.

E-mail addresses: vvgrushin@mail.ru (V.V. Grushin), dobr@ipmnet.ru (S.Yu. Dobrokhotov), SergeevSe1@yandex.ru (S.A. Sergeev).
} 